\title{
Type 2 diabetes: unravelling the interaction between genetic predisposition and lifestyle
}

\author{
W. Rathmann • B. Kowall • G. Giani
}

Received: 9 May 2011 / Accepted: 31 May 2011 /Published online: 28 June 2011

(C) Springer-Verlag 2011

Keywords Genetics · Gene-lifestyle interaction · Lifestyle . Type 2 diabetes

\author{
Abbreviations \\ EPIC European Prospective Investigation into Cancer \\ and Nutrition \\ SNP Single-nucleotide polymorphism
}

The high concordance rate of type 2 diabetes in monozygotic twins, the increased risk of diabetes in the offspring of affected parents and the difference in the prevalence of diabetes between various ethnic groups suggest a high heritability for this disorder [1]. On the other hand, modern societies are characterised by intense urbanisation, which is linked to tremendous environmental and behavioural changes: physical inactivity, an energy-dense diet, psychosocial stress and exposure to environmental pollutants [1-3]. The descriptive epidemiology of type 2 diabetes suggests that the interaction between genetic susceptibility and these modern lifestyle factors, as well as environmental risk factors, is critical to the development of the disease [1].

Current case-control studies have succeeded in identifying dozens of novel risk variants (see Herder and Roden [1] and the references therein), but have failed to explain most of the expected genetic contribution to the risk of type 2 diabetes [4]. Thus, there is a need for

W. Rathmann $(\bowtie) \cdot$ B. Kowall $\cdot$ G. Giani

Institute of Biometrics and Epidemiology,

German Diabetes Centre,

Auf'm Hennekamp 65,

40225 Duesseldorf, Germany

e-mail: rathmann@ddz.uni-duesseldorf.de additional study designs to address the issue of 'missing heritability'. This missing heritability might at least partly be due to the presently unknown modifying effect of lifestyle factors, and therefore adequately powered epidemiological studies to investigate gene-lifestyle interaction in the prediction of type 2 diabetes are both timely and welcome [5].

A major challenge is that dietary patterns and physical activity are not easy to assess in epidemiological studies [6]. Although a number of scientific consortia have been established to undertake meta-analyses of genetic data from loci associated with type 2 diabetes, different definitions and measurements of diet and physical activity hamper the pooling of such data to investigate gene-lifestyle interactions $[1,6]$. The study of these complex and multidimensional behaviours ideally requires protocols in which the standardisation of instruments has already been implemented at the design stage [7].

The European Prospective Investigation into Cancer and Nutrition (EPIC) was initiated in the late 1980s and involved standardised baseline information on lifestyle behaviours [8]. The goal of the InterAct consortium is to carry out a meta-analysis of gene-lifestyle interactions in terms of the risk of type 2 diabetes based on EPIC cohorts from eight European countries [9].

\section{Strengths and weaknesses}

One strong advantage of InterAct is the huge database $(12,403$ incident cases of type 2 diabetes identified among 340,234 EPIC participants). Thousands of individuals with incident type 2 diabetes are usually required for a prospective study of gene-environment interactions even 
to detect moderate interactions with common gene variants [6], and InterAct will most probably have this power. Another strength of InterAct is the nested case-cohort design (including a centre-stratified subcohort of 16,154 individuals), which confines the assessment of genetic data to a small part of the whole study group and, at the same time, offers the advantages of a prospective study.

Health behaviour, in particular related to diet and physical activity, was assessed in a standardised manner across Europe. This allows a pooling of detailed data on dietary self-reports, developed and validated within each of the eight participating countries and including an assessment of nutritional biomarkers [9]. Standardising and validating the procedure to assess exposure to these factors provides a more accurate estimate of the true underlying relationship between an exposure and an outcome. In the EPIC Study, considerable work was carried out to make sure that the repeatability and validity of the self-reported measures were at an acceptable level. However, food frequency and physical activity questionnaires always have limitations because food intake and physical activity are complex and variable over time so that they are difficult to recall and to report with the desired precision.

One drawback is that InterAct was planned retrospectively to study type 2 diabetes. Therefore, in the single EPIC studies, the methods used to ascertain and verify diabetes vary and include self-reported diabetes, diagnosis by medication, doctor-diagnosed diabetes and registerbased identification of individuals with diabetes. The high-sensitivity approach used to identify diabetes in InterAct maximises statistical power, but the heterogeneity of methods to ascertain the condition may have an influence on the study results:

- The frequency of alleles associated with an increased risk of diabetes may depend on the method of ascertaining the diabetes. As an example, the gene encoding transcription factor 7-like 2 (TCF7L2) has been identified as a type 2 diabetes locus by genome-wide linkage studies, and it has been suggested that the polymorphism affects the capacity of pancreatic beta cells to secrete insulin $[1,4]$. In line with this finding, the risk-carrying $\mathrm{T}$ allele of TCF7L2 single-nucleotide polymorphism (SNP) rs7903146 was over-represented in individuals requiring insulin treatment and under-represented in the patients managed by diet alone [10]. Since in some EPIC centres identification of cases was mainly based on medication for diabetes (insulin, oral glucose-lowering agents), the risk-carrying T allele of this TCF7L2 SNP and possibly SNPs in other genes may be associated with the definition of type 2 diabetes within the InterAct population. This may bias apparent associations between genes and the presence of diabetes.
- Given that the disease characteristics vary with the method used to ascertain diabetes, it is possible that the gene-environment interaction is not the same when diabetes is, for example, ascertained by self-report or by assessing the intake of drugs to treat diabetes. Thus, the bias in estimating the interaction depends on the proportion of diabetes identified by self-report or by medication from within the whole study group.

Furthermore, the lack of glucose measurements is a drawback of InterAct. As an example, given the high prevalence of undiagnosed type 2 diabetes [11], it is unclear how many of the 1,058 individuals with incident diabetes in the first and second year of follow-up had prevalent diabetes and should therefore have been excluded from the analyses [9]. There is also a potential for underestimating the incidence of type 2 diabetes due to the large number of individuals whose diabetes can only be detected by fasting blood glucose or oral glucose tolerance tests. In fact, the overall incidence in InterAct was 3.76 per 1,000 person-years, which is lower than is currently estimated in glucose-based European cohort studies in adults (with a range of 8 to 19 per 1,000 person-years) [12].

Generally, InterAct comprises heterogeneous populations with different methods of ascertaining their diabetes (see above), with different age ranges and sex distributions [9]. Furthermore, the recruitment strategies of the EPIC cohorts varied widely [9]. Thus, it will be important to evaluate whether the results are consistent across studies. This will help to put into context how generalisable the results are. Finally, the baseline data were collected between 1993 and 1998; therefore there needs to be consideration of how patterns of environmental risk factors may have changed since then.

\section{Conclusion and outlook}

The InterAct project is a valuable resource for prospectively investigating the effect of the interaction between behavioural and genetic factors on the risk of type 2 diabetes across Europe. The results, when available, will help to provide individually tailored prevention strategies for people who have an increased risk of developing diabetes. In addition, the unique database may help to provide new insights into the interaction between biomarkers and genetic variants. The InterAct database will contain known and novel biomarkers representing intermediate pathways, i.e. inflammatory markers and markers of liver and kidney function, iron status and lipid metabolism [9]. Biomarkers of blood or genetic variants cannot currently improve the accuracy of traditional risk factors for type 2 diabetes in predicting the risk of the disorder [13]. 
InterAct will have sufficient power to help to explain the interaction between biomarkers and lifestyle factors on the incidence of type 2 diabetes. This improvement of risk prediction for type 2 diabetes is crucial for the identification of high-risk individuals who could benefit from targeted prevention [13].

Acknowledgements W. Rathmann and G. Giani are supported by the Federal Ministry of Health (Berlin, Germany) and the Ministry of Innovation, Science, Research and Technology of the state of NorthRhine Westphalia (Duesseldorf, Germany). W. Rathmann and B. Kowall are supported by a grant from the German Federal Ministry of Education and Research (BMBF) within the GANI MED (Greifswald Approach to Individualized Medicine) project (Ernst-Moritz-ArndtUniversität Greifswald). The authors thank C. Herder (German Diabetes Center) for contributions to parts of the commentary.

W. Rathmann drafted the article, and B. Kowall and G. Giani critically revised it. All authors have approved the final version for publication.

Duality of interest The authors declare that there is no duality of interest associated with this manuscript.

\section{References}

1. Herder C, Roden M (2010) Genetics of type 2 diabetes: pathophysiologic and clinical relevance. Eur J Clin Invest 41:679-692

2. Tamayo T, Herder C, Rathmann W (2010) Impact of early psychosocial factors (childhood socioeconomic factors and adversities) on future risk of type 2 diabetes, metabolic disturbances and obesity: a systematic review. BMC Public Health 10:525
3. Krämer U, Herder C, Sugiri D et al (2010) Traffic-related air pollution and incident type 2 diabetes: results from the SALIA cohort study. Environ Health Perspect 118:1273-1279

4. McCarthy MI (2011) Dorothy Hodgkin Lecture 2010. From hype to hope? A journey through the genetics of type 2 diabetes. Diabet Med 28:132-140

5. Franks PW, Mesa JL, Harding AH, Wareham NJ (2007) Genelifestyle interaction on risk of type 2 diabetes. Nutr Metab Cardiovasc Dis 17:104-124

6. Wareham NJ, Young EH, Loos RJF (2008) Epidemiological study designs to investigate gene-behavior interactions in the context of human obesity. Obesity 16(Suppl 3):S66-S71

7. Palla L, Higgins JP, Wareham NJ, Sharp SJ (2010) Challenges in the use of literature-based meta-analysis to examine geneenvironment interactions. Am J Epidemiol 171:1225-1232

8. Riboli E, Hunt KJ, Slimani N et al (2002) European Prospective Investigation into Cancer and Nutrition (EPIC): study populations and data collection. Public Health Nutr 5:1113-1124

9. The InterAct Consortium (2011) Design and cohort description of the InterAct Project: an examination of the interaction of genetic and lifestyle factors on the incidence of type 2 diabetes in the EPIC Study. Diabetologia. doi:10.1007/s00125-011-2182-9

10. Kimber CH, Doney AS, Pearson ER et al (2007) TCF7L2 in the Go-DARTS Study: evidence for a gene dose effect on both diabetes susceptibility and control of glucose levels. Diabetologia 50:1186-1191

11. Rathmann W, Haastert B, Icks A et al (2003) High prevalence of undiagnosed diabetes mellitus in Southern Germany: target populations for efficient screening. The KORA survey 2000. Diabetologia 46:182-189

12. Rathmann W, Strassburger K, Heier M et al (2009) Incidence of type 2 diabetes in the elderly German population and the effect of clinical and lifestyle risk factors: KORA S4/F4 cohort study. Diabet Med 26:1212-1219

13. Herder C, Karakas M, Koenig W (2011) Biomarkers for the prediction of type 2 diabetes and cardiovascular disease. Clin Pharmacol Ther. doi:10.1038/clpt.2011.93 